\title{
La justicia de paz en una provincia del interior argentino (Mendoza, 1872-1890)*
}

\section{Magistracy in a Province of the Argentine Interior (Mendoza, 1872-1890)}

\author{
Eliana Fucili \\ (D) https://orcid.org/0000-0002-2508-5202 \\ Facultad de Derecho \\ Universidad Nacional de Cuyo, Argentina \\ elianafucili@gmail.com
}

\section{David Terranova \\ (D) https://orcid.org/0000-0001-6988-1027 \\ Facultad de Derecho \\ Universidad Nacional de Cuyo \\ Facultad de Ciencias Empresariales y Jurídicas \\ Universidad Juan Agustín Maza, Argentina \\ davidgterranova@gmail.com}

Resumen: Este trabajo busca realizar un aporte a la historia social de la justicia a través de enfoques y metodologías sujetos a la actual agenda de investigación sobre las instituciones estatales. Puntualmente, se propone presentar un análisis sobre cómo estaba organizada la justicia de paz en el territorio mendocino entre 1872-1890, un periodo en el que se dictaron las primeras leyes

* Agradecemos a los evaluadores de este trabajo sus comentarios y sugerencias que han contribuido a mejorar algunos aspectos del análisis.

Cómo citar: Fucili, E. y Terranova, D. (2020). La justicia de paz en una provincia del interior argentino (Mendoza, 1872-1890). Secuencia (108), e1743. DoI: https://doi.org/10.18234/secuencia.v0i108.1743

cc $(1)$ Esta obra está protegida bajo una Licencia Creative Commons Atribución-NoComercial 4.0 Internacional. 
que regularon la administración de la justicia baja en la provincia. En efecto, durante esos años la fisonomía del poder judicial local adquirió mayor cohesión como resultado del desarrollo político e institucional del poder central y provincial. Asimismo, el trabajo centra su atención en quiénes fueron los actores que ejercieron los fueros de paz en la provincia, atendiendo principalmente, a sus perfiles socioocupacionales.

Palabras clave: justicia; magistrados territoriales; Mendoza; siglo xıx; leyes.

Abstract: This article seeks to contribute to the social history of justice through approaches and methodologies linked to the current research agenda on state institutions. Specifically, it attempts to analyze the way magistracy in Mendoza was organized between 1872-1890, the period when the first laws regulating the administration of low justice in the province were enacted. Indeed, during those years, the local judiciary acquired greater cohesion because of the political and institutional development of the central and provincial power. The article also focuses on the actors who exercised magistracy in the province, mainly exploring their sociooccupational profiles.

Key words: justice; territorial magistrates; Mendoza; 19th century; laws.

Recibido: 6 de mayo de 2019 Aceptado: 11 de octubre de 2019

Publicado: 12 de agosto de 2020

L

a construcción del orden político decimonónico y la organización del - poder institucional debieron asentarse en una extensa ramificación territorial, en la centralización de mecanismos de ejercicio del poder y en el desarrollo de nuevos medios de coacción social y control (Fradkin, 2007, pp. 9-23). Estas cuestiones han sido recorridas por diversas líneas historiográficas que han profundizado en el análisis de cómo se conformó y organizó el poder en los diversos espacios provinciales, atendiendo a sus propias problemáticas internas y en relación con otros espacios locales. Entre las diversas líneas interpretativas surgidas en las últimas décadas -al calor de la renovación de la historia política, social y de la justicia-, una serie de estudios ha utilizado diversos recursos y herramientas metodológicas que han permitido restituir 
evidencia empírica sobre la composición y funcionamiento de la baja justicia en diferentes espacios regionales. ${ }^{1}$

Tomando como punto de partida estas investigaciones, el presente trabajo propone indagar en una escala local las formas de organización de la administración de la justicia de paz o baja justicia en el territorio de Mendoza durante las últimas décadas del siglo xIx, a fin de realizar un aporte a la historia social de la justicia y a la actual agenda de investigación sobre las instituciones estatales. Para ello, se realizará un análisis sobre la organización institucional de la justicia de paz en el territorio mendocino entre 1872-1890, centrando la mirada en los actores políticos y sociales que intervinieron en ese fuero judicial.

Conviene advertir que estas cuestiones no han pasado desapercibidas entre los estudios locales de la provincia de Mendoza, que han presentado restituciones institucionales y normativas acerca de la conformación del poder judicial (Ábalos, 2009; Egües, 2008; Seghesso de López Aragón, 1997) y han avanzado recientemente en el tratamiento de los agentes locales de la baja justicia: jueces de paz, decuriones y comisarios. Estos últimos trabajos han presentado caracterizaciones de perfiles socioeconómicos del personal administrativo en la primera mitad del siglo xix (Molina, 2008); y de la conformación de los jueces de paz en la provincia, atendiendo a sus antecedentes jurídicos, funciones y competencias (Sanjurjo de Driollet, 2003). En líneas generales, dichas investigaciones han señalado ciertas continuidades en relación con la organización y administración de justicia entre la época colonial y los años siguientes al colapso del imperio español -que han quedado plasmadas en algunos rasgos que adquirió la fisonomía judicial en los estados provinciales y, más tarde, en la Constitución Nacional dictada en 1853-, aunque también han destacado rupturas, resemantizaciones o nuevos usos de modalidades institucionalistas heredadas de la monarquía española (Molina, 2018).

El arco temporal escogido abarca desde 1872, fecha en la que se dictó la primera Ley Orgánica del Poder Judicial, hasta 1890. Su selección se fundamenta en que permite realizar un primer acercamiento a nuestro objeto de estudio y examinar la sanción de las leyes que organizaron el sistema judicial local $(1872,1876$ y 1880) y la puesta en marcha, en el corto plazo, de la estructura

${ }^{1}$ Al respecto, la bibliografía es abundante; por ello, sólo señalaremos algunos trabajos que han constituido interesantes puntos de partida para esta investigación: Garavaglia (1997, pp. 241-262; 1999); Gelman (2000, pp. 7-31); Fradkin (2007); Barriera (2009, 2010); Molina (2010); Caselli (2016); Yangilevich (2017, pp. 73-94); Corva (2014); entre otros. 
judicial-administrativa en lo referente a los nombramientos de jueces de paz. Durante esos años, sostenemos, la fisonomía del poder judicial local adquirió mayor cohesión como resultado del desarrollo político e institucional del poder central y provincial. Es decir, las leyes sancionadas buscaron, al igual que en otras provincias, regular el sistema judicial en el marco de la organización territorial y administrativa de la provincia; $y$ de los preceptos enarbolados por la Constitución Nacional (1853) y la Carta Magna Provincial (1854).

Atendiendo a estas cuestiones, este trabajo propone avanzar sobre dos aspectos: ¿cómo se produjo la conformación y estructuración de la baja justicia en Mendoza? y ¿quiénes compusieron el cuerpo de jueces de paz en la provincia? El primero de ellos se focaliza en la puesta en marcha de la organización de la justicia de paz: el propósito de su regulación, las funciones designadas a las nuevas autoridades judiciales y el contexto político-institucional en el que se dictaron. Para ello se consultarán las leyes relativas a la organización del poder judicial en lo que respecta a la justicia territorial $(1872,1876$ y 1880) con el propósito de reconstruir el esquema judicial. El segundo aspecto por indagar busca reconstruir las características socioocupacionales de los actores políticos y sociales que se desempeñaron en esa instancia judicial y su inserción en la política departamental y/o provincial. Estas cuestiones se analizarán a partir de herramientas que brinda la prosopografía, las cuales contribuyen a describir y caracterizar el perfil de un actor colectivo y permiten examinar el background de los individuos que conforman el corpus de estudio (Stone, 2011). Por otro lado, se incorporarán perspectivas metodológicas de los análisis sociográficos que brindan valiosas herramientas para comprender la composición de un elenco político, sus relaciones, prácticas en uso y sus trasformaciones en el tiempo (Ferrari, 2012).

Para abordar los perfiles de los actores judiciales territoriales, se confeccionó una base datos que permite realizar tratamientos cuantitativos y cualitativos. Dicha base reúne información de 108 jueces de paz, referidas a datos biográficos (nacimiento, lugar de procedencia, profesión/oficio, entre otros) y trayectorias político-administrativas. Esta información ha sido recabada a partir del rastrillaje de un nutrido corpus documental que incluyó los Registros Oficiales de la Provincia de Mendoza y los Partes Departamentales. Estos permitieron reconstruir el entramado judicial e identificar la inserción político-administrativa del actor. Asimismo, se recurrió a la consulta de los censos nacionales de 1869 y 1895 a fin de recaudar información individual sobre el mayor número de los jueces de paz registrados. 


\section{ALGUNAS CONSIDERACIONES EN TORNO A LA ORGANIZACIÓN DE LA BAJA JUSTICIA EN MENDOZA}

Desde los primeros años del proceso revolucionario, los comisarios y decuriones estuvieron a cargo del ejercicio de la justicia inferior (Molina, 2008). Tras la aprobación del Reglamento de Policía de 1828 aumentaron sus atribuciones de policías, referidas a la vigilancia y control -matricular a la población de su cuartel, mantener el control de la movilidad de sus habitantes, "controlar vagos y malentretenidos"- y al cuidado del aspecto material de su jurisdicción -abrir y cerrar hijuelas de aguas, reparar puentes, conservar acequias, entre otros-. Además, dichos funcionarios tuvieron competencia en causas menores, similares a las que, hasta entonces, habían ejercido los alcaldes de barrio y los alcaldes de hermandad durante el periodo colonial (Molina, 2010; Sanjurjo de Driollet, 2004). Mientras los comisarios ejercían esas funciones en los departamentos, los decuriones lo hacían en los cuarteles (distritos administrativos en que se dividía la ciudad y los departamentos), constituyéndose ambos en una pieza fundamental del poder gubernamental ya que eran las autoridades más cercanas a la población y podían mediar entre ella y el gobierno. De hecho, su inserción y sus vínculos con la comunidad local eran los principales elementos que tenía en cuenta el poder ejecutivo provincial a la hora de designarlos en tales cargos, a propuesta del jefe de policía de la capital o los subdelegados en la campaña o de la municipalidad (Molina, 2010).

Más tarde, los comisarios y decuriones recibieron nuevas atribuciones con el dictado del Reglamento de Estancia de 1834, que fijó su actuación como jueces de conciliación. Dichas atribuciones fueron ejercidas hasta 1846, cuando se creó un Juzgado de Conciliación que funcionó hasta 1859, fecha en que se devolvieron esas facultades a los comisarios y decuriones. Unos años después, la Ley Orgánica del Poder Judicial (1872) les retiró las facultades judiciales a estos magistrados, que, en adelante, serían agentes del ejecutivo y policía municipal, cuyos nombramientos corresponderían al ejecutivo provincial a propuesta de la municipalidad. ${ }^{2}$ Asimismo, dicha ley buscó sentar las bases para la organización de la justicia provincial que quedó conformada por el Superior Tribunal de Justicia y los juzgados inferiores.

${ }^{2}$ Véase al respecto: Reglamento de Estancia, 1834 y Ley Orgánica del Poder Judicial, 1872. Sección Independiente. Archivo General de la Provincia de Mendoza (en adelante AGPM), Argentina. 
El primero estaba compuesto de tres jueces, que debían ser mayores de 30 años, abogados y poseer la ciudadanía en ejercicio. La ley ponía énfasis en la separación de los poderes del Estado y especificaba que quedaban excluidos de dicho cuerpo colegiado los empleados del poder ejecutivo y del legislativo. De esta manera, se incorporaba el principio de incompatibilidad para ejercer ambas funciones. La estructura judicial se completaba con los juzgados inferiores, compuestos por: jueces de letras -uno civil y otro criminal-y jueces de paz. ${ }^{3}$ Los de letras debían ser nombrados por el poder ejecutivo provincial $y$ duraban cinco años en el ejercicio de sus funciones y gozaban de una renta mensual fija. ${ }^{4}$ Para ejercer dicho cargo se requería ser abogado, mayor de 25 años y con ciudadanía en ejercicio. ${ }^{5}$ La ley preveía -al igual que para el Superior Tribunal de Justicia- la exclusión de dichas funciones para aquellos que tuvieran empleo en el poder ejecutivo y/o el legislativo. Sin embargo, en 1874, dicha cláusula fue modificada a fin de permitir que tanto los jueces de letras como los miembros del Superior Tribunal de Justicia, pudieran ejercer, en simultáneo, cargos en la legislatura mendocina, debido a la escasez de letrados provinciales. ${ }^{6}$ Por entonces, la provincia contaba, según el censo nacional de 1869, con nueve abogados en todo su territorio. ${ }^{7}$

Por su parte, los jueces de paz que integraban los juzgados inferiores debían distribuirse de la siguiente manera: dos en la ciudad, uno por cada distrito; y uno en cada uno del resto de los departamentos de campaña. Cada uno de ellos contaría con un juez de paz suplente para reemplazarlo en caso de ausencia o legítimo impedimento. ${ }^{8}$ La creación de los jueces de paz en la provincia tuvo como principal objetivo reorganizar y equilibrar los poderes de los funcionarios territoriales. Tal como se mencionó con anterioridad, el nuevo funcionario de la baja justicia quitó las atribuciones judiciales a los comisarios y decuriones; así como también a los subdelegados, quienes hasta entonces concentraban en sus manos facultades ejecutivas y judiciales. Su sanción fue precedida por amplios debates en el recinto legislativo y entre

3 Véase Ley Orgánica del Poder Judicial, 1872, art. 1.

${ }^{4}$ Véase Ley Orgánica del Poder Judicial, 1872, arts. 17, 20, 21 y 22.

5 Véase Ley Orgánica del Poder Judicial, 1872, art. 23.

${ }^{6}$ Véase Ley del 11 de marzo de 1874, art. 1 que deroga los art. 23 y 34 de la ley orgánica de tribunales.

${ }^{7}$ Datos extraídos del Censo Nacional de 1869. Disponible en http://www.deie.mendoza. gov.ar

${ }^{8}$ Véase Ley Orgánica del Poder Judicial, 1872, art. 2. 
la opinión pública local (Sanjurjo de Driollet, 2003). Fundamentalmente, la discusión rondó en torno a dos posturas: unos sostenían que el ejercicio de la justicia territorial era atribución exclusiva de las municipalidades, fundamentando su postura en la Constitución Provincial de 1854; mientras que otros apelaban a la división de poderes para argumentar que dichos fueros recaían en el ámbito exclusivo del poder judicial y no en una institución que cumpliera funciones ejecutivas.

Años más tarde, la ley de 1872 fue modificada en dos oportunidades. La primera en 1876, luego de que la legislatura mendocina sancionara una ley en donde determinaba la necesidad de reformar los aspectos estructurales y procesales de la justicia de paz. ${ }^{9}$ Dicha reforma tuvo como objetivo principal definir y aumentar las atribuciones de los jueces de paz, a fin de garantizar el ejercicio de la justicia territorial con mayor autonomía respecto de la figura del subdelegado, cuya función, dos años antes había ganado mayor peso político-administrativo en las municipalidades producto de la reforma de la Ley de Municipalidades (Pérez Guilhou, 1997).

La segunda modificación legislativa fue en 1880, cuando el gobernador interino, Nicolás Godoy, justificó a partir de un diagnóstico de la realidad judicial, la necesidad de modificar la forma de concebir la administración de la baja justicia. ${ }^{10}$ Godoy postulaba que en un pueblo democrático todos los ciudadanos debían ser iguales ante la ley; sin embargo, la realidad provincial daba cuenta de que la baja justicia no estaba al alcance de todos, ya que era para unos pocos una actividad meramente lucrativa, inmoral y que su amparo no alcanzaba a los pobres, sino sólo a aquellos que pudieran afrontar los costos que la justicia demandaba. Asimismo, el poder ejecutivo provincial consideraba que obligar y someter a un procedimiento formulista y complicado a los jueces que administraban justicia en los asuntos de ínfima cuantía, implicaba desconocer y dar por tierra con los principales fundamentos de la justicia de paz: la cercanía del juez con el conflicto y las partes y la celeridad en la resolución de los casos. ${ }^{11} \mathrm{Al}$ respecto, Godoy argumentó frente a la legislatura mendocina la reforma de la siguiente manera:

9 Véase Ley del 23 de marzo de 1876, art. 1.

${ }^{10}$ Nota del gobernador Nicolás Godoy a la Legislatura con motivo de la presentación del proyecto de reforma de la justicia de paz, septiembre de 1879. Extraído de Registro Oficial de la Provincia de Mendoza, 1880, p. 249.

${ }^{11}$ Registro Oficial de la Provincia de Mendoza, 1880, p. 250. 
El Proyecto del Ejecutivo tiende a descentralizar la administración de la justicia inferior; a convertir en realidad el precepto constitucional que estatuye la descentralización de todos los poderes del Gobierno. Nuestra vida municipal está aún en embrión, y debemos ir alimentándola con cautela, para que no ponga en jaque a las autoridades políticas, no para que caiga en la inercia por su propia impotencia. Es preciso no olvidar que el unitarismo tiene, entre nosotros, raíces de siglos, y que el federalismo es de ayer; y este último sistema de Gobierno, si no se arregla bien, es capaz para todo lo malo e inhábil para lo bueno. ${ }^{12}$

Ahora bien, en términos generales, ¿cuáles fueron los requisitos para acceder al ejercicio de la justicia de paz? La ley de 1872 estipulaba que debía ser mayor de 22 años; saber leer y escribir; tener ciudadanía en ejercicio y renta propia o una profesión honorable. No podían ejercer las funciones de jueces de paz quienes fueran empleados públicos, abogados con estudio abierto, escribanos o personas con oficio o funciones que tengan conexión con la administración de justicia. ${ }^{13} \mathrm{Su}$ nombramiento correspondía a las municipalidades o, en caso de no existir aún dicha institución, dependía del poder ejecutivo provincial con acuerdo de la legislatura mendocina. Cada juez de paz duraba un año en sus funciones y podía ser reelegido. ${ }^{14}$ Era un cargo obligatorio y "nadie podía excusarse de aceptarlo sino por justas casusas que debía evaluar la municipalidad y en su defecto el Poder Ejecutivo". ${ }^{15}$

La modificación realizada en 1876 no introdujo reformas al respecto y, en términos generales, ambas normas buscaban separar la función judicial de la ejecutiva en el territorio, aunque era la municipalidad la que designaba al juez de paz. Sin embargo, la reforma introducida en 1880 cambió esta concepción y amplió la cantidad de actores intervinientes: mantuvo la figura del Juez de Paz y devolvió funciones judiciales a los comisarios y decuriones. De esta manera, la nueva ley buscó fragmentar la competencia de cada uno de estos actores y jerarquizar su función al determinar la forma de apelación de sus resoluciones y, por lo tanto, la importancia en la pirámide institucional.

\footnotetext{
${ }^{12}$ Registro Oficial de la Provincia de Mendoza, 1880, p. 250

${ }^{13}$ Véase Ley Orgánica del Poder Judicial, 1872, art. 11.

14 Véase Ley Orgánica del Poder Judicial, 1872, art. 12.

15 Véase Ley Orgánica del Poder Judicial, 1872, art. 10.
} 
En lo referido a las competencias de los jueces de paz, la ley de 1872 estipuló que podía entender acciones en materia civil y comercial que no excediera de 150 pesos; acciones que surjan producto de los contratos de alquiler; las cuestiones relativas a los ajustes y obligaciones respectivas de los jornaleros y demás trabajadores y los que los emplean; las acciones civiles por calumnias, injurias verbales e injurias escritas que no se den en el ámbito de la prensa; las causas de hurtos simples. A estas funciones, los jueces de paz departamentales sumaban la relativa a los juicios sucesorios, quedando excluidos de la órbita de los jueces de paz de la capital. Por su parte, la reforma de 1876 amplió la jurisdicción, ya que, de ahora en más, resolverían casos civiles de hasta 200 pesos, siendo prorrogable hasta 1000 pesos por el consentimiento de las partes. En materia sucesoria, se mantuvo la diferenciación presente entre los jueces de paz de los departamentos y los de la capital. La última modificación legislativa analizada hacia 1880, fraccionaba aún más la competencia según el monto del pleito. Por lo tanto, los decuriones entenderían en casos de hasta seis pesos, los comisarios conocerían en juicios desde seis y hasta 20 pesos. Por último, los jueces de paz decidirían en casos desde 20 y hasta 200 pesos, siendo prorrogable su jurisdicción hasta 1000 pesos con consentimiento de ambas partes, así como también en toda la materia que había sido concedida en la ley anterior.

Una vez producida la resolución judicial, la legislación estableció de manera diversa la fuerza de la validez de la decisión, así como también la forma de revisión de esta. La primera ley analizada determinaba que las sentencias de los jueces de paz causarían ejecutoria, siempre que la cantidad no excediese de 25 pesos. En caso de que así ocurriera, habría apelación ante tres vecinos del distrito que debían nombrar las partes o el juez de paz, si estos no lo hicieran dentro del tercer día. En relación con las sentencias que pronunciasen los jueces de paz en asuntos criminales, serían siempre apelables en la forma en que se establece en los juicios civiles. De esta manera, el establecimiento de estos jurados para la apelación significaba asegurar la justicia de paz dentro de la órbita municipal e implicaba un principio de descentralización judicial. Por su parte, la modificación legislativa de 1876 aumentó el carácter ejecutorio de las sentencias pronunciadas por los jueces de paz, dado que se constituyeron en obligatorias $y$, por lo tanto, exigibles, siempre y cuando no excedieran de 30 pesos del principal o no fuesen apeladas dentro del término legal. Cuando el valor del pleito sobrepasara lo especificado, se determinaba que podía apelarse ante un Tribunal de Distrito, compuesto por 
dos vocales y un presidente. La diferencia en relación con la ley anterior radicó en que, en este caso, constituyó una atribución exclusivamente del juez de paz la selección de esos miembros, de una lista de ocho ciudadanos por cada juzgado que formarán al principio de cada año las municipalidades.

Por último, en 1880 se estipuló un procedimiento de apelación más jerarquizado, garantizando el principio del juez natural, en función de la diversificación de actores judiciales intervinientes. Las resoluciones de los decuriones podían apelarse dentro del tercer día ante el comisario más cercano, mientras que, respecto de la resolución del comisario en grado de apelación, no existía recurso alguno. Por su parte, las resoluciones de los comisarios en primera instancia serían apelables ante el juez de paz más cercano. El Tribunal de Apelación debía estar compuesto por tres vecinos (y tres suplentes), designados a principio de cada año por las municipalidades, para conocer en las apelaciones de las sentencias de los jueces de paz. De esta forma, la composición del Tribunal de Apelación quedaba totalmente fuera de la órbita de los jueces de paz y, por lo tanto, del poder judicial; mientras que la municipalidad, representante del poder ejecutivo local, era la que determinaba su conformación con total discrecionalidad vulnerando el principio de división de poderes.

Respecto a la remuneración que percibían las autoridades de la baja justicia mendocina, la ley de 1872 -al igual que la ley de 1876- estipulaba que no tendrían un salario fijo otorgado por el Estado provincial ni por la municipalidad, sino que debían ser retribuidos por la parte condenada, dependiendo de la cuantía del juicio. ${ }^{16}$ Por lo tanto, el pago al juez seguía la misma suerte que la ejecución de la sentencia; es decir, que no solamente le bastaba con terminar el juicio para cobrar, sino que dependía de que el vencido tuviera sustento económico para hacer frente a ambas deudas, la de la causa objeto del juicio y la del emolumento de quien administraba justicia. Este punto fue fuertemente cuestionado por quienes impulsaron la reforma de 1880, dado que favorecía la parcialidad del juez a la hora de resolver sus sentencias. Por ello, la ley de 1880 estipuló que el sueldo de los jueces de paz debía ser pagado por la municipalidad o el poder ejecutivo según corresponda, sin poder ser disminuido. Dicha reforma fue un elemento crucial para asegurar la imparcialidad de los jueces territoriales en la resolución de los pleitos y la indepen-

${ }^{16}$ Ley Orgánica del Poder Judicial de 1872, art. 15, y Ley Orgánica y de Procedimientos de la Justicia de Paz de 1876, art. 42. 
dencia respecto de los demás poderes del Estado, al consagrar el principio de intangibilidad de las remuneraciones. ${ }^{17}$

\section{¿QUIÉNES FUERON LOS JUECES DE PAZ QUE ADMINISTRARON LA JUSTICIA MENOR EN EL TERRITORIO MENDOCINO?}

Tal como se señaló en la introducción, el rastrillaje realizado sobre la documentación oficial conservada permitió confeccionar una base de datos que reúne información sobre 108 individuos que fueron designados jueces de paz en el territorio provincial entre 1872 y 1890. Es interesante aclarar que, si bien la legislación vigente a partir de 1880 contemplaba la ampliación del personal destinado a ejercer la justicia de paz, se ha tomado como criterio de análisis la inclusión únicamente de los jueces de paz a fin de trazar una caracterización general de estos actores políticos y sociales que tuvieron un fuerte protagonismo en el ejercicio de la baja justicia.

A partir de la información recolectada es posible someter la base de datos al análisis de algunas variables que permitan presentar evidencia empírica sobre las trayectorias de los individuos que desempeñaron los cargos de jueces de paz y sus respectivos perfiles socioocupacionales. En primer lugar, interesa indagar ¿cómo fueron las trayectorias públicas de los jueces de paz? y ¿cuál fue su proyección en la política departamental y/o provincial? A fin de presentar un primer acercamiento al problema, se confeccionó la gráfica 1 , que permite visualizar la cantidad de veces que ejercieron el cargo de juez de paz los actores estudiados; y a partir de ello detectar los itinerarios individuales y colectivos.

De acuerdo con los datos arrojados, se observa la relativa movilidad del personal de la baja justicia mendocina. En efecto, alrededor de $82 \%$ de los individuos registrados en la base de datos ejerció entre una y dos veces el cargo de juez de paz, coincidiendo que $50.5 \%$ de ellos fue el único cargo político-administrativo que ejerció; mientras que el resto de ellos (49.5\%), además de las funciones de justicia de paz, ejercieron cargos de menor jerarquía dentro de

${ }_{17}$ Conviene advertir que dicho principio implementado en la baja justicia mendocina fue receptado en la reforma constitucional provincial de 1895. Véase, al respecto, a Seghesso de Lopez Aragón (1997). 


\section{Gráfica 1. Cantidad de veces que un individuo ejerció el cargo de juez de paz}

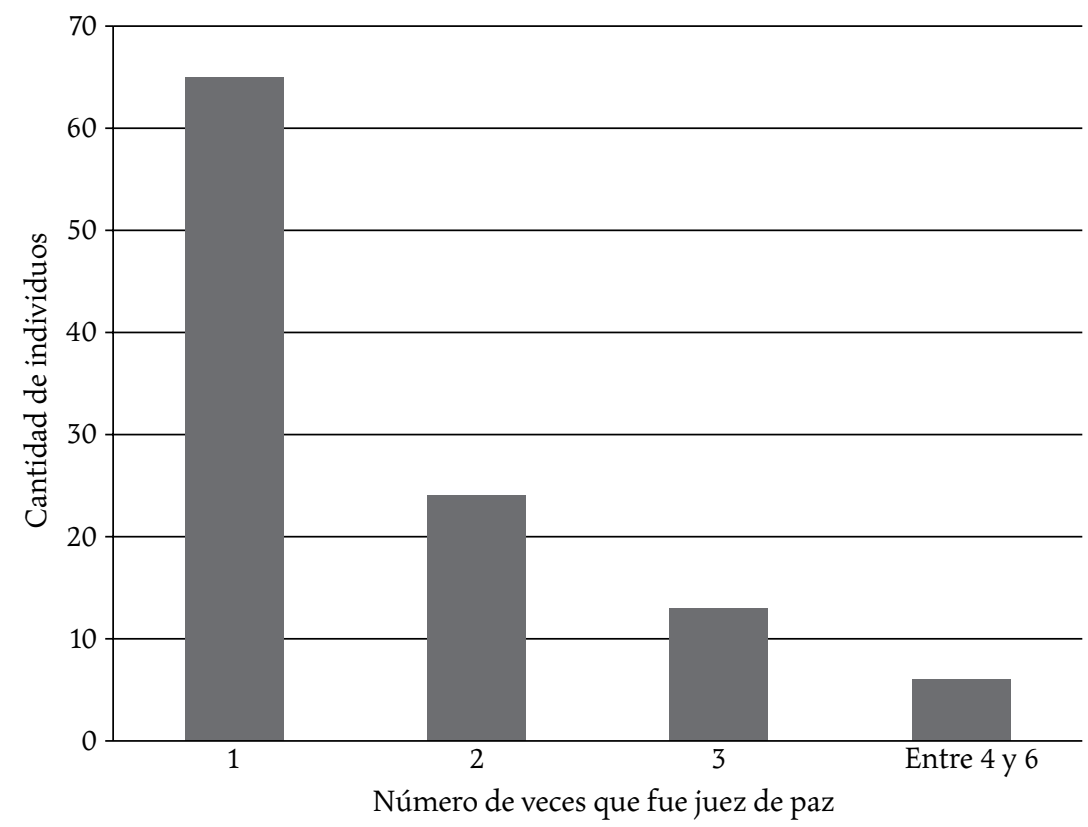

Nota: Gráfica confeccionada con base en los 108 individuos que componen la base de datos de esta investigación.

Fuente: elaboración propia con base en la consulta del Registro Oficial de la Provincia de Mendoza (Mendoza, 1852-1890); Carpetas departamentales (Mendoza, 1850-1890). Sección Independiente. AGPM, Argentina.

los límites de la jurisdicción departamental donde desempeñaron funciones, tales como encargado de mesa electoral, comisario, decurión y, en menor medida, cargos de relieve en el poder ejecutivo departamental. Por su parte, $17 \%$ de los 108 individuos estudiados ejerció entre tres y seis veces la función de juez de paz, desarrollando, casi en su totalidad, trayectorias político-administrativas de relieve en las jurisdicciones departamentales y/o desempeñando funciones políticas en la provincia. 
La observación de estas primeras cifras hace posible conjeturar la moderada proyección que tuvieron los magistrados de la baja justicia territorial en la política departamental y provincial. En efecto, se ha contabilizado que únicamente 39 (36.1\%) de los 108 individuos estudiados tuvieron trayectorias de mayor relieve. Conviene advertir que, de ellos, 32 se posicionaron como referentes territoriales, es decir que contaban con cierto arraigo y reconocimiento entre sus vecinos. Tal como ha expuesto Garavaglia para la experiencia de la campaña bonaerense, tales condiciones resultaron necesarias para el desempeño de las funciones propias de la baja justicia y un elemento importante en la construcción de la legitimidad necesaria. Una lectura atenta de sus itinerarios individuales indica que 21 de ellos fueron nombrados subdelegados de campaña, máxima magistratura rural cuya designación anual era exclusiva competencia del gobernador de la provincia y recaía sobre actores políticos y sociales de las jurisdicciones que debían administrar. Los once restantes fueron electos concejales municipales, cargo creado por la Ley de Municipalidad de 1868 que estipulaba la existencia de un cuerpo colegiado para administrar las jurisdicciones departamentales, aunque las sucesivas reformas realizadas en la década de 1870 lo colocaron en un escalafón jerárquico por debajo del subdelegado (Sanjurjo de Driollet, 2004).

Asimismo, se observa que, de los 39 individuos con mayor peso político-administrativo, 22 traspasaron los límites departamentales y se posicionaron en las arenas políticas provinciales: $95.4 \%$ de ellos participó al menos una vez en la legislatura provincial; $22.7 \%$ ejerció funciones en el poder judicial de Mendoza y $13.6 \%$ fue designado -al menos una vez-como miembro del concejo de gobierno.

En términos generales, se observa que $85 \%$ de las trayectorias político-administrativas departamentales y/o provinciales de estos individuos se iniciaron antes de su designación como juez de paz. Es decir, el desempeño de este cargo no implicó ningún tipo de ascenso o descenso en sus itinerarios. Un caso emblemático fue el de Donato Guevara (1819-1882), un comerciante mendocino, vecino de la villa sureña de San Carlos. Los datos recogidos sobre sus pasos por la vida política departamental y provincial indican que Guevara era un actor político de segunda línea en su jurisdicción. Sus primeros pasos en la vida pública local fueron como escrutador de mesas electorales instaladas en dicho departamento entre finales de la década de 1850 y la década siguiente. En esa misma villa ejerció la máxima magistratura rural -subdelegado- en reiteradas oportunidades, primero de forma interina (en- 
tre 1862 y 1865) y luego como propietario (1869-1870). Asimismo, fue incorporado como miembro de numerosas comisiones organizadas por el Estado provincial destinadas a atender necesidades locales. Puntualmente, Guevara fue miembro de la comisión destinada a inspeccionar la educación (1870); consignar denuncias y avalúos de propiedades raíces (1871); regular los bienes raíces departamentales (1872), entre otras. En 1873, apenas puesta en vigencia la Ley Orgánica de Justicia, fue nombrado juez de paz de San Carlos, cargo que ejerció hasta 1874, cuando fue electo diputado provincial. Al terminar su mandato en la legislatura, en 1876, Guevara fue designado nuevamente como juez de paz de su jurisdicción, cargo que ocupó de forma consecutiva hasta 1882, cuando falleció.

Por otro lado, $15 \%$ de los jueces restantes ejercieron dicha magistratura al comienzo de sus trayectorias públicas, lo que permite conjeturar sobre las posibilidades políticas que pudo brindarles el ejercicio de la justicia de paz territorial para luego acceder a cargos de mayor relevancia. Sin embargo, conviene advertir que los individuos identificados bajo esta condición tuvieron trayectorias públicas efímeras y de poco alcance político y social. En ese sentido, resulta ilustrativo el breve itinerario público de Manuel Ventura, un hacendado del departamento de San Rafael que dio sus primeros pasos públicos como juez de paz en su departamento (1874-1876) y luego accedió a una banca en la legislatura (1876-1878). Al finalizar dicha función, se retiró del ejercicio de cargos político-administrativos y, probablemente, continuó abocado a su oficio.

Ahora bien, ¿cuál fue el peso que tuvo la condición de vecino en la designación de los jueces de paz departamentales? A través de los datos recabados en los censos nacionales analizados, ha sido posible reconstruir información sobre 65 (60.1\%) de los 108 individuos que componen el universo de estudio de esta investigación. En primer lugar, la comparación de los datos sobre la jurisdicción donde residían los funcionarios con los del departamento en que desempañaron funciones de juez de paz, permite advertir que 30 individuos ejercieron cargos de la baja justicia departamental en la jurisdicción donde eran vecinos; mientras que los 35 individuos restantes lo hicieron en departamentos en los que no eran vecinos. Al respecto, la legislación vigente no tenía ninguna especificación sobre algún requisito respecto a que los jueces de paz debieran residir en la jurisdicción donde ejercieran el cargo. En realidad, la importancia de la cercanía de la justicia de paz con el conflicto estuvo dada por la instalación del juzgado mismo en la jurisdicción en la cual el pleito se produce. 
Otra variable sociográfica que resulta de interés indagar se refiere al perfil ocupacional. De acuerdo con la pesquisa realizada en los censos, se ha podido trazar el perfil de 60 de los 108 individuos estudiados. Conviene advertir que dicha información resulta parcial, ya que sólo aporta datos relativos a la profesión/oficio de los actores estudiados, pero no define la magnitud de las propiedades o comercios, ni los niveles de estudios alcanzados. No obstante, nos permiten realizar una mirada general sobre los perfiles de los jueces de paz mendocinos.

En referencia a la alfabetización, $100 \%$ de los jueces censados declaró saber leer y escribir, un requisito fundamental para el desempeño de la justicia territorial. En cambio, sus perfiles ocupacionales fueron mucho más heterogéneos. A través de la gráfica 2 , es posible ilustrar la información recabada. En primer lugar, se observa que $61 \%$ de los jueces de paz censados declararon ser propietarios y comerciantes; $18 \%$ poseer perfiles especializados en saberes administrativo-técnicos o título profesional; $18 \%$ desarrollar oficios rurales y el 3\% restante pertenecer al sector artesano.

Es decir, el mayor porcentaje de los jueces de paz analizados pertenecía a los sectores propietarios y comerciantes de la provincia, los cuales controlaban los principales mecanismos del comercio de exportación e importación; y en términos generales tenían una mayor injerencia en las arenas políticas locales y/o nacionales (Fucili, 2019). En efecto, 35\% de los propietarios y comerciantes que ejercieron el cargo de juez de paz realizó trayectorias políticas departamentales y provinciales de mayor relieve. Por su parte, en relación con los individuos que declararon ser "empleados de gobierno" o poseer algún título profesional, se han contabilizado en total a once individuos, de los cuales nueve eran empleados de gobierno; es decir, a partir del ejercicio de cargos públicos habían desarrollado cualidades para el manejo de los asuntos político-administrativos; mientras que entre los dos restantes se cuenta un abogado y un agrimensor. De ellos, $80 \%$ alcanzó puestos político-administrativos de mayor relevancia a lo largo de sus trayectorias. Finalmente, se distinguen aquellos jueces de paz que declararon desempeñar oficios rurales en el interior provincial, es decir, peones, jornaleros y labradores; y quienes realizaban tareas artesanales: talabartero, carpinteros, entre otros. En términos generales, estos personajes con perfiles socioocupacionales de baja calificación no desarrollaron trayectorias públicas notorias ni prolongadas en el tiempo, sino más bien se caracterizaron por el desempeño de uno o dos cargos públicos en toda su trayectoria. 


\section{Gráfica 2. Perfil ocupacional de los jueces de paz mendocinos (1872-1890)}

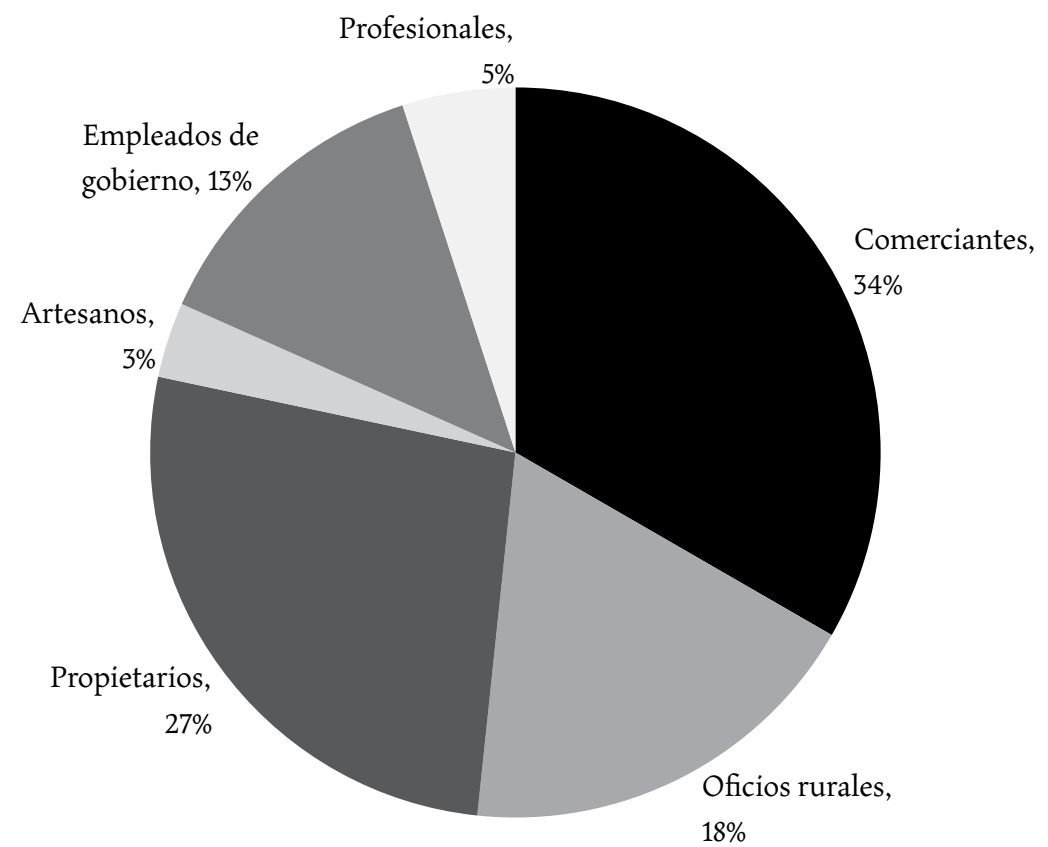
nacionales.

Nota: Gráfica confeccionada con base en 60 jueces de paz localizados en los censos y 1895.

Fuente: elaboración propia con base en la consulta de los Censos Nacionales de 1869

Estas breves pinceladas sobre los perfiles de los jueces territoriales mendocinos guardan ciertos puntos de comparación con sus homónimos del interior de otras provincias, tales como Entre Ríos y Buenos Aires. Al respecto, los estudios realizados por Pressel han subrayado que el perfil de los jueces de campaña de Uruguay y Concordia (en Entre Ríos) se caracterizó por la preeminencia de actores abocados a labores vinculadas al mundo rural: la mayoría de ellos eran propietarios con diferentes niveles de fortuna y dependientes de cierto rango (encargados y capataz) (Pressel, 2009). Para Buenos Aires, los estudios pioneros de Garavaglia (1997), Fradkin (2007) y Yangilevich (2017) 
han subrayado que en el interior de la provincia los agentes de la baja justicia de campaña contaban con perfiles ocupacionales abocados a oficios rurales y comerciales con patrimonios medianos y pequeños.

\section{CONSIDERACIONES FINALES}

El breve recorrido realizado en estas páginas ha ofrecido los primeros resultados sobre el tratamiento, relativamente exhaustivo, de la baja justicia mendocina entre 1872 y 1890 . En su conjunto, el examen realizado ha permitido presentar algunos aspectos poco documentados con base en la organización de la justicia territorial y en la caracterización de los perfiles de quienes ejercieron dichas funciones. En relación con el primero de ellos, el análisis de la legislación vigente por entonces $(1872,1876$ y 1880$)$ ha posibilitado reconstruir un cuadro sobre la puesta en funcionamiento de la justicia de paz instaurada en la provincia, en un clima de organización y crecimiento estatal. La puesta en funcionamiento de la maquinaria judicial en el territorio provincial buscó, por un lado, respetar la división de poderes en las jurisdicciones departamentales al quitarle atribuciones judiciales a los subdelegados $\mathrm{y}$; por otro, fragmentar las competencias de los funcionarios territoriales a fin de jerarquizar la administración de la justicia de menor cuantía.

Asimismo, el análisis presentado permite restituir evidencia sobre las trayectorias individuales del cuerpo de funcionarios estudiados y trazar algunas características generales. En primer lugar, ha sido posible distinguir un núcleo de funcionarios -aproximadamente 36\% de los estudiados- que realizó trayectorias de mayor relieve, logrando afianzar su poder territorial $y$, en algunos casos, traspasar las arenas departamentales y ejercer magistraturas provinciales. El resto de los jueces de paz estudiados no desarrollaron trayectorias político-administrativas de relevancia, por el contrario, tuvieron un escaso manejo de la política departamental y/o provincial, lo que explica la relativa movilidad del personal que ejercía las funciones de justicia territorial.

Finalmente, la evidencia reunida ha permitido delinear los perfiles ocupacionales de los jueces de paz mendocinos y distinguir, entre ellos, la preponderancia de los sectores propietarios y comerciantes. Estos individuos contaron con mayores posibilidades para realizar trayectorias políticas de mayor alcance, frente a otros personajes con capitales ocupacionales más precarios -los trabajadores rurales y sectores de artesanos- que no lograron 
posicionarse, en la misma proporción, en las arenas políticas departamentales y/o provinciales. En su conjunto, los rasgos de sus perfiles ocupacionales permiten conjeturar sobre la baja calificación técnica del cuerpo de jueces de paz de la provincia, ya que sólo aproximadamente $18 \%$ de ellos había realizado trayectorias administrativas en el aparato burocrático estatal en formación, mientras que el resto probablemente debió adquirir los saberes necesarios a partir del ejercicio de los cargos administrativos.

\section{LISTA DE REFERENCIAS}

Ábalos, M. (2009). La Constitución de Mendoza, comentarios históricos, normativos y jurisprudenciales. En Constituciones provinciales y de la ciudad de Buenos Aires comentadas. Buenos Aires: Abeledo Perrot.

Barriera, D. (comp.) (2009). Justicia y fronteras. Estudios sobre la historia de la justicia en el Río de la Plata. Siglos XVI-XIX. Murcia: Universidad de Murcia.

Barriera, D. (2010). La justicia y las formas de la autoridad. Organización política y justicia local en territorios de frontera. El Río de la Plata, Córdoba, Cuyo y Tucumán, siglos XVIII y XIX. Rosario: ISHIR/CONICET-Red Columnaria.

Caselli, E. (coord.) (2016). Justicia, agente y jurisdicciones. De la monarquía hispánica a los Estados nacionales (España y América, siglos XVI-XIX). Madrid: Fondo de Cultura Económica.

Corva, M. (2014). Constituir el gobierno, afianzar la justicia: el poder judicial de la provincia de Buenos Aires 1853-1881. Rosario: Prohistoria.

Egües, C. (2008). Historia constitucional de Mendoza. Los procesos de reforma. Mendoza: EDIUNC.

Ferrari, M. (2012). Acerca del abordaje sociográfico de los elencos políticos, sus prácticas y autorrepresentaciones. Algunas reflexiones. PolHis. Boletín Bibliográfico Electrónico del Programa Buenos Aires de Historia Política, 10, 241-250. Recuperado de http://polhis.com.ar/archivo/polhis10/

Fucili, E. (2019). Forjadores del poder. Retratos de dirigentes territoriales de Mendoza en la segunda mitad del siglo xIx. Mendoza: EDIUNC.

Fradkin, R. (2007). El poder y la vara: estudios sobre la justicia y la construcción del Estado en el Buenos Aires rural: 1780-1830. Buenos Aires: Prometeo.

Garavaglia, J. C. (1997). Paz, orden y trabajo en la campaña: la justicia rural y los juzgados de paz en Buenos Aires, 1830-1852. Desarrollo Económico, 37(146), 241-262. 
Garavaglia, J. C. (1999). La justicia rural en Buenos Aires durante la primera mitad del siglo XIX (Estructuras, funciones y poderes locales). En Poder, conflicto y relaciones sociales. El Río de la Plata, XVIII-XIX. Rosario: Homo Sapiens.

Gelman, J. (2000). Crisis y reconstrucción del orden en la campaña de Buenos Aires. Estado y sociedad en la primera mitad del siglo xix. Boletín del Instituto de Historia Argentina y Americana Dr. Emilio Ravignani, 21, 7-31. Recuperado de http://ravignani.institutos.filo.uba.ar/publicacion/n\%C2\%BA-21-enero-junio-2000

Molina, E. (2008). Justicia y poder en tiempos revolucionarios: las modificaciones en las instituciones judiciales subalternas de Mendoza (1810-1820). Revista de Historia del Derecho, 35.

Molina, E. (2010). Los funcionarios subalternos de justicia en Mendoza, 1820-1852: entre el control comunitario y el disciplinamiento social. Nuevo Mundo Mundos Nuevos. DoI: https://doi.org/10.4000/nuevomundo.59353

Molina, E. (2018). Modalidades de espacialización política: de la justicia de proximidad a otras prácticas de agencia gubernamental en Barriales, jurisdicción de Mendoza (1814-1850). En Justicias situadas, entre el virreinato rioplatense y la República Argentina (1776-1864). La Plata: Universidad Nacional de La Plata.

Pérez Guilhou, D. (1997). Instalación del régimen municipal en Mendoza. En Ensayos sobre la historia política institucional de Mendoza. Buenos Aires: Secretaría Parlamentaria, Comisión de Cultura, Dirección de Publicaciones.

Pressel, G. E. (2009). Los hombres que administran la justicia local. La persistencia de la notabilidad en el oriente entrerriano (1841-1853). En D. Barriera (comp.), Justicia y fronteras. Estudios sobre la historia de la justicia en el Río de la Plata. Siglos XVIXIX, Murcia: Universidad de Murcia.

Sanjurjo de Driollet, I. (2003). La justicia de paz en Mendoza. Leyes de 1872, 1876 y 1880. Revista Confluencia, 1(2), 129-143.

Sanjurjo de Driollet, I. (2004). La organización político-administrativa de la campaña mendocina en tránsito del antiguo régimen al orden liberal. Buenos Aires: Instituto de Investigaciones de Historia del Derecho.

Seghesso de López Aragón, M. C. (1997). Historia constitucional de Mendoza. Mendoza: Instituto Argentino de Estudios Constitucionales y Políticos.

Stone, L. (2011). Prosopografía. Reista. Sociologia e Política, 19(39), 115-137.

Yangilevich, M. (2017). Justicia de paz y organización del territorio en la campaña sur de Buenos Aires durante la primera mitad del siglo xIx. Un diálogo con Juan Carlos Garavaglia. Prohistoria, 28, 73-94. Recuperado de http://www.scielo.org.ar/ scielo.php?script=sci_abstract\&pid=S1851-95042017000200004 